\title{
Simulation Study on Modeling and Operation Characteristics of Lubricating System of Marine Power Plant
}

\author{
Caofengshou Xiong ${ }^{+}$, He Ni, Yanqiao Chen and Jiashan Jin \\ College of Power Engineering, Naval University of Engineering, Wuhan 430033 ,China
}

\begin{abstract}
In order to deeply study the operating characteristics of the lubricating oil system of marine power plant, the flow resistance, node pressure and auxiliary calculation modules are constructed based on the flow, pressure and heat transfer characteristics of the system components with the fluid network modular modeling method. Based on the analysis of the structural characteristics and working principle of a certain lubricating oil system of marine power plant, a dynamic simulation model of the lubricating oil system coupled with flow and heat transfer is established by means of the SimuWorks simulation platform. According to the actual test procedure, the main working parameters of the lubricating oil system in the typical dynamic process such as start-stop, switching and system changing conditions of the lubrication oil pump are calculated. which proves a strong basis for system control strategy optimization, and has certain engineering value.
\end{abstract}

Keywords: marine power plant; lubricating system; modular modeling; dynamic simulation

\section{Introduction}

The lubricating oil system is an important auxiliary system for the marine power system. Its operating state largely determines the stability and safety of the power system, affecting directly the reliability and service life of the power unit. Therefore, in the research of the operation characteristics of the lubricating oil system, the optimization of the control strategy and the diagnosis of the system, it is necessary to have a good command of the parameters of lubricating parts of the power unit under different working conditions such as the lubrication oil pressure, flow rate and temperature, etc .

Lubricating oil system simulation is one of the important means to obtain the lubricating oil's flow parameters of the system. The relevant researches and applications are very extensive [1-4]. Based on the mathematical model of the oil pipeline, nozzle and bearing, Chun S M [5] established a simulation model that can be used for the analysis of the engine lubricating oil system. It also used the model to carry out the system flow distribution and pressure distribution simulation. Moussa Nahim H [6] studied and established a simulation program analyzing comprehensively the system fault of the marine diesel engine lubricating oil system. Through simulation experiments, a database applicable to system fault diagnosis and fault-tolerant control was obtained through simulation experiments. Su Lichao [7] used Flowmaster to simulate the lubricating oil pressure of the fuel supply subsystem of the aero-engine lubrication system, and obtained the distribution of the oil pressure inside the fuel supply system under steady state conditions.

The majority of the above researches are based on subsystems. Most of the simulation calculations are carried out for a specific working condition, and they generally do not consider the heat exchange between the lubricating oil and the external environment during its flow in the pipeline. This obviously does not truly reflect the flow characteristics of the lubricating oil system. Therefore, in this paper, a dynamic simulation model of a certain marine power plant lubricating oil system is established basing on the SimuWorks system simulation platform. The factor of the system components'size and the coupling factors of the lubricating oil

\footnotetext{
+ Corresponding author. Tel.: + 8618108674502.
}

E-mail address: xcfs81@163.com. 
flow and heat transfer are considered. Also, based on the operating characteristics of the actual trial run, the typical processes of the lubricating oil pump, such as start-stop, switch and system variable working conditions, are dynamically simulated. By analyzing the change of parameters concluding pressure and temperature of each key node during the operation of the system, the key factors that affect the dynamic response of the system are summarized, and the optimization strategy is given. The research results can be used as the basis for system design, and have certain engineering application value.

\section{Introduction to the lubricating system of a type of marine power plant}

The lubricating oil system of the marine power plant consists of a lubricating oil tank, a steam turbine oil pump, an electric oil pump, a lubrication oil cooler, a lubricating oil filter, a lubricating oil pipeline, a flow control valve, etc. The operating principle of the oil system is shown in Figure 1.

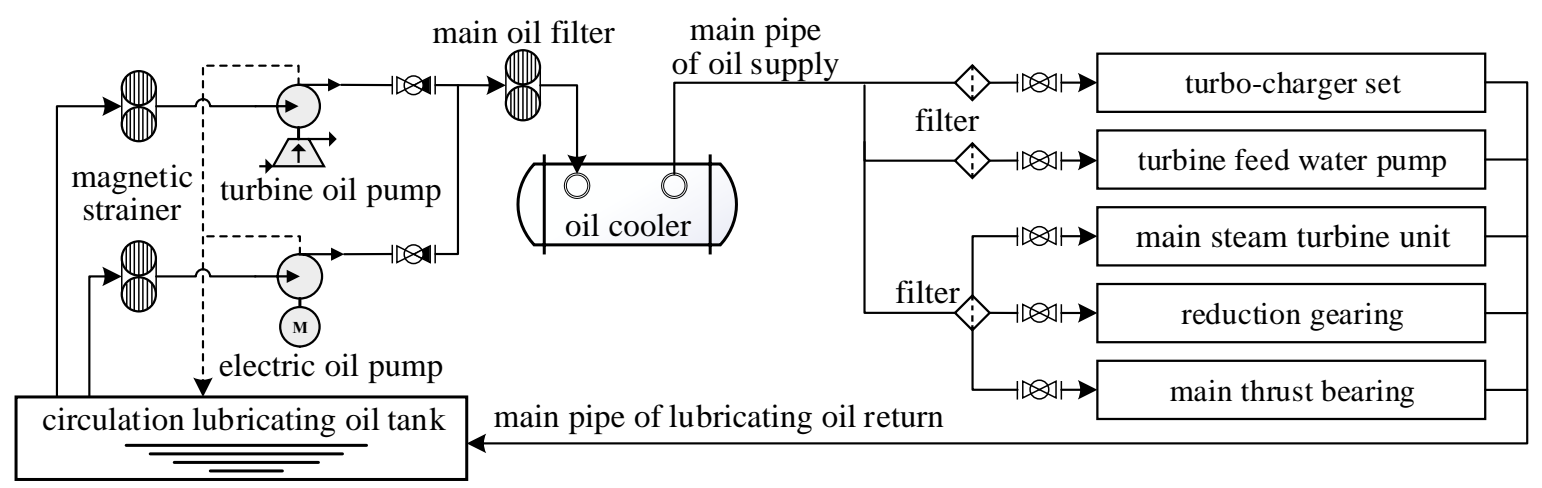

Fig 1: Schematic diagram of a marine power plant oil system

\section{Modular modeling of oil system}

\subsection{Module division}

The marine oil system is a typical fluid network system in which a pipeline system consisting of components such as pipes and valves connects the various oil equipment. Because the topology of the lubricating oil system of the marine power plant is more complicated, this paper uses the modular modeling idea [8-9] to analyze this fluid network. According to the functional characteristics of each component of the lubricating oil system, the composition of whole network is divided into three categories: flow resistance type, node pressure type and auxiliary type module.

\subsection{Module model}

\subsubsection{Flow resistance model}

The flow resistance model includes all resistive components through which the oil flows in the system, such as: oil filters, oil coolers, oil lines, flow control valves, etc. The control body is divided by the inner wall surface of each component flow channel and the area surrounded by the inlet and outlet end faces. When the oil is used for one-dimensional flow, the momentum conservation equation is:

$$
\frac{L_{f}}{A_{f}}\left(\frac{\partial W_{f}}{\partial t}+W_{f} \frac{\partial W_{f}}{\partial L}\right)=P_{f 1}-P_{f 2}+\rho_{f} g \Delta H_{f}-\frac{f_{w}}{A_{f}}
$$

Where, $W_{f}$ is the average mass flow rate of the fluid; $P_{f 1}$ and $P_{f 2}$ is the pressure of the fluid at the inlet and outlet of the module; $\Delta H_{f}$ is the elevation difference of the inlet and outlet of the module; $L_{f}$ is the length of the flow channel; $A_{f}$ is the average cross-sectional area of the flow channel; $\rho_{f}$ is the density of the oil.

Ignore the difference between the module's import and export heights, which is $\Delta H_{f}=0$, and take into account $\partial W_{f} / \partial L=0$ on the premise of the piecewise lumped parameters. According to the modeling criteria, the oil in the flow resistance model module is treated as an incompressible fluid, which is $\partial W_{f} / \partial t=0$. The flow rate and the pressure of the module at this time -- have approximate square root relationships:

$$
W_{f}=C_{f} \sqrt{\left|\Delta P_{f}\right|}
$$

Where, $\Delta P_{f}=P_{f 1}-P_{f 2}$ is the inlet and outlet pressure difference; $C_{f}$ is the total admittance coefficient, 
reflecting the flow capacity of the flow channel.

Since the flow of the oil in the flow channel does not undergo a phase change, it is stable turbulence when $\Delta P_{f}$ is greater than the transition pressure difference $\Delta P_{g}$, and is laminar flow when $\Delta P_{f}$ is less than the transition pressure difference $\Delta P_{g}$. Therefore, when the simulation step size is small enough, the equation (2) can be linearized [9-10]:

$$
\left\{\begin{array}{l}
W_{f}=B_{t} \Delta P_{f} \\
W_{f}=B_{c} \Delta P_{f} / \Delta P_{g}
\end{array}\right.
$$

Where, $B_{t}$ is the turbulent linear admittance coefficient; $B_{c}$ is the laminar linear admittance coefficient.

The lubricating oil exchanges with the environment during the flow of the oil in the flow channel, and the amount of heat exchange is related to the degree of heat insulation of the flow channel. The amount of heat exchange $Q_{1}$ and $Q_{2}$ between the oil and the pipe wall and the pipe wall and the environment can be approximately calculated by the following formula [11]:

$$
\left\{\begin{array}{l}
Q_{1}=\left(\lambda_{f}+\alpha_{1} W_{f}^{0.8}\right) S_{1}\left(\frac{T_{f 1}+T_{f 2}}{2}-\bar{T}_{f p}\right) \\
Q_{2}=\alpha_{2} S_{2}\left(\bar{T}_{f p}-T_{0}\right)
\end{array}\right.
$$

Where, $\bar{T}_{f p}$ is the average temperature of the composite wall; $h_{f 1}$ and $h_{f 2}$ are the specific enthalpy of the inlet and outlet oils; $\lambda_{f n}$ is the thermal conductivity of the oil; $T_{f 1}$ and $T_{f 2}$ are the temperature of the inlet and outlet oils; $T_{0}$ is the ambient temperature; $\alpha_{1}, \alpha_{2}$ and $S_{1}, S_{2}$ are respectively coefficient of heat transfer and heat exchange area of the inner and outer surfaces of the composite wall.

\subsubsection{Node pressure model}

The node pressure model includes the inlet and outlet of each device, the pipeline port and interface, and the pressure measurement point. Considering the compressibility of the fluid, a control body with a certain volume is selected according to the flow boundary of the oil, and the size should be such that the internal parameters can be treated as lumped parameters, thereby establishing a equation of mass conservation:

$$
V_{p}\left(\frac{\partial \rho_{f}}{\partial P_{p}} \frac{\partial P_{p}}{\partial t}+\frac{\partial \rho_{f}}{\partial T_{p}} \frac{\partial T_{p}}{\partial t}\right)=\sum_{i=1}^{n} W_{f i}-W_{p e}
$$

Where, $V_{p}$ is the volume of the control body for the node; $\rho_{f}$ is the density of the oil; $T_{p}$ is the temperature of the node; $P_{p}$ is the pressure of the node; $W_{f i}$ is the mass flow into or out of the node of the $\mathrm{i}$-th flow resistance module; $W_{p e}$ is the leakage flow of the node.

Since the fluid pressure changes faster than the temperature, it can be considered that $\partial T_{p} / \partial t=0, W_{f i}$ can be obtained by the formula (3), the formula (5) can be simplified into [12]:

$$
K_{N P S} \frac{d P_{p}}{d t}=\sum_{i=1}^{n} B_{i}\left(P_{i}-P_{p}\right)-W_{p e}
$$

Where, $P_{i}$ is the upstream or downstream pressure of the node; $B_{i}$ is the linear admittance of the corresponding flow; $K_{N P S}=V_{p} \partial \rho_{f} / \partial P_{p}$ is the compression coefficient of the fluid.

\subsubsection{Oil pump model}

There are two oil pumps in the lubricating oil system of the marine power plant, which are turbine oil pump and electric oil pump respectively. The turbine oil pump is composed of an auxiliary steam turbine, a reducer and a screw pump. The electric oil pump is composed of an electric motor and a screw pump. The pump bodies of the two oil pumps are the same and share one pump module.

(1) Turbine rotor module

The module input is the power working on the rotor. From the conservation of energy, the dynamic equation of the rotor is [13]:

$$
\frac{d n_{a x}^{2}}{d t}=\frac{P_{q l c}-P_{l o s s}-P_{w}}{J}
$$


Where, $n_{a x}$ is the rotor speed; $P_{\text {loss }}$ is the rotor loss power; $P_{w}$ is the power consumption of the oil pump; $\mathrm{J}$ is the total moment of inertia.

(3) Motor module

The electric oil pump is driven by an electric motor whose speed is fixed. Therefore, the module adopts linear increment or decrement to simulate the start or stop of the motor. The calculation formula is:

$$
\frac{d N_{g}}{d t}=\Delta N_{g}
$$

Where, $N_{g}$ is the speed; $\Delta N_{g}$ is a given speed increment.

(4) Oil pump body module

In the lubricating oil system, the oil pumps are all screw pumps. The pump's structural parameters, pump speed and oil inlet and outlet pressure are used as input to the module. The calculation formula between the flow $W_{p}$ and the power consumption $P_{w}$ is:

$$
\begin{gathered}
W_{p}=60 V_{0} \rho_{f} \frac{n_{p}}{n_{0}} \\
P_{w}=\frac{W_{p}\left(P_{p 1}-P_{p 2}\right)}{1000 \rho_{f}}
\end{gathered}
$$

Where, $W_{p}$ is the mass flow of the output oil for the pump; $V_{0}$ is the volume of the pump; $\rho_{f}$ is the density of the oil; $n_{p}$ is the speed of the pump; $n_{0}$ is the number of turns required to transfer the $V_{0}$ volume of oil to the screw pump; $P_{w}$ is the power consumed by the pump; $P_{p 1}$ and $P_{p 2}$ are the pressure of the inlet and outlet oil of the pump.

\subsection{System model}

This paper uses the SimuWorks simulation platform developed by Beijing Dafeng Tianli Technology Co., Ltd. to build a dynamic simulation model of the marine power plant's lubricating oil system. The model includes 49 modules, as shown in Figure 2.

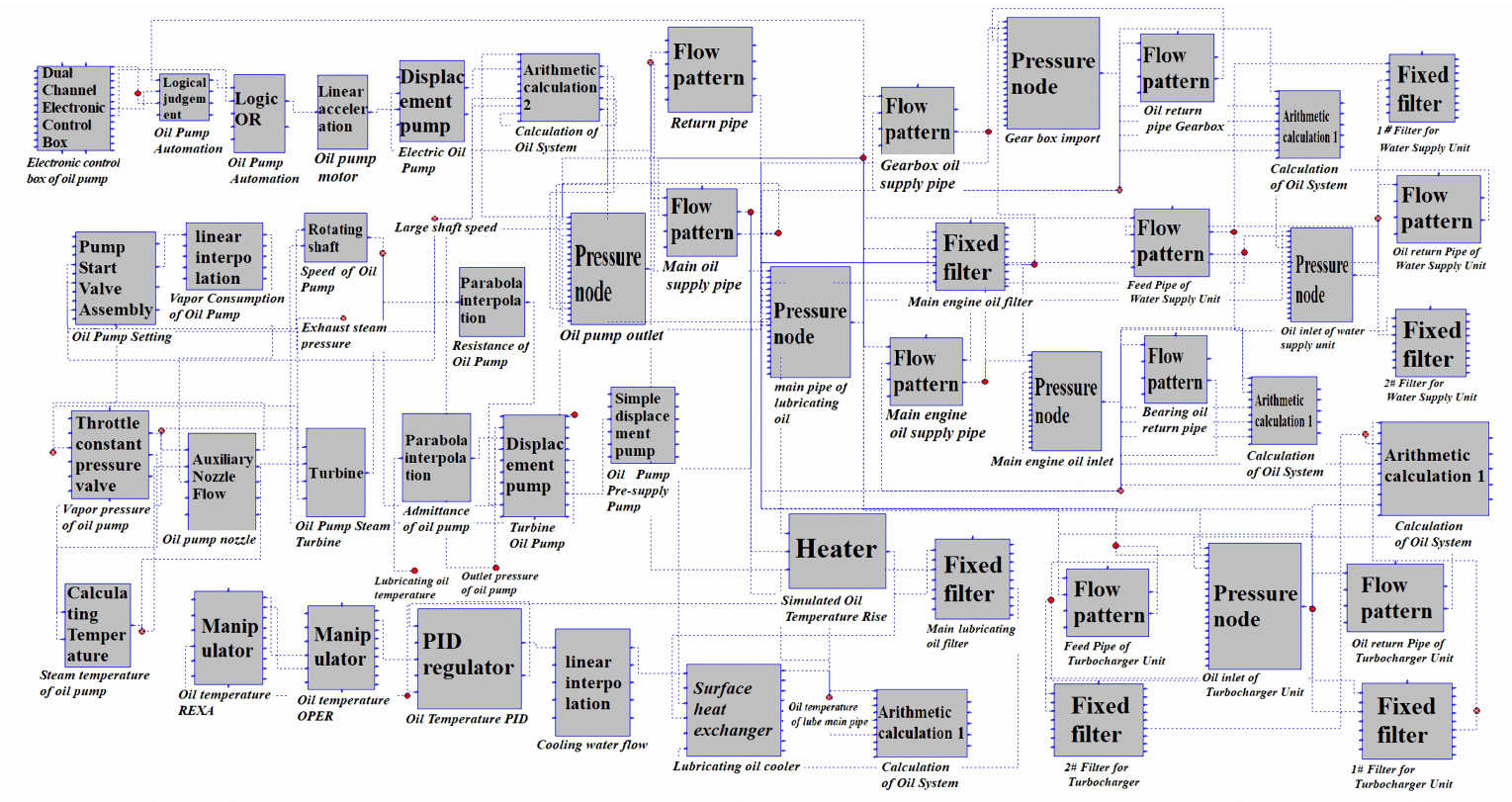

Fig 2: Simulation calculation model of the marine power plant's lubricating oil system

\section{Simulation study on the operating characteristics of the lubricating oil system}

In order to verify the difference between the simulation results of the model and the actual operating parameters during the dynamic operation of the system, and to study the operating characteristics and performance status of each component and the whole system, this paper selects the key operations that determines the system's state according to the actual test procedure of the oil system of the marine power 
plan and reasonably splits and combines them. Three dynamic simulation experiments are conductedrespectively the "electric oil pump start-up system was upgraded to a low working condition process", "the steam turbine oil pump start-up system was raised to a high working condition process" and "oil temperature and dynamic change process of regulating valve opening". The parameters of oil pressure $(P)$, turbine pump speed $(r)$ and oil temperature $(\mathrm{T})$ are treated by dimensionless method. The results are as follows.

\subsection{Dynamic simulation of the starting process of electric oil pump}

In order to study the starting characteristics of the electric oil pump and the response of the oil pressure of each key equipment in the process from the start-up to the low working condition, the process that the electric oil pump is started alone in the shutdown state of the oil system is selected as the simulation test process. According to the structural characteristics of the electric oil pump, the motor is linearly accelerated, and the rated speed is established in only 0.6 seconds, so the delay for start-up and starting to establish the pressure could be neglected.

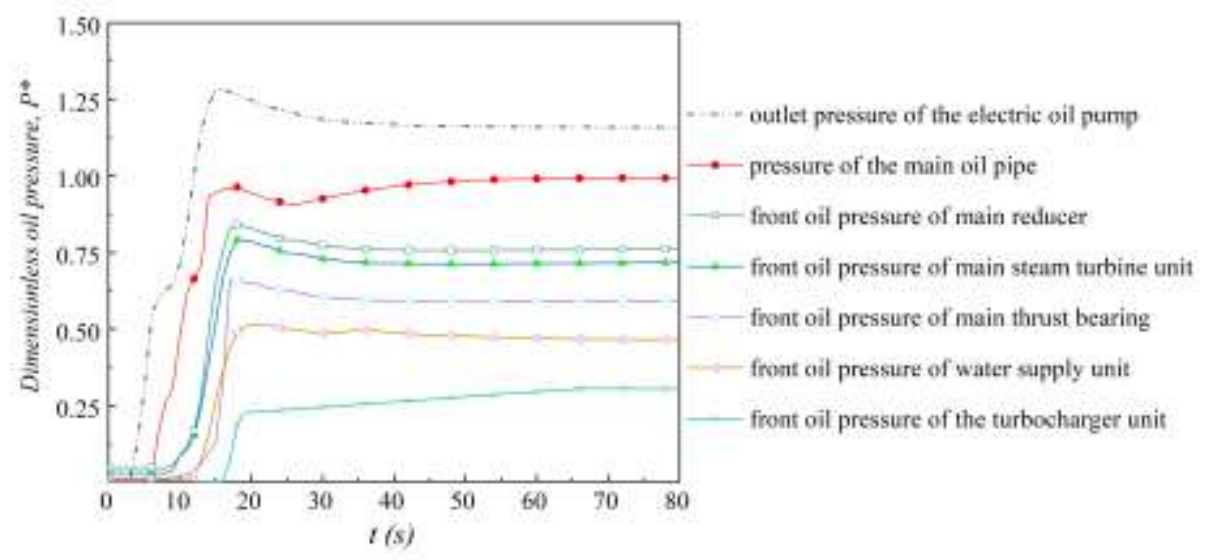

Fig 3: Changes in various parameters during the start-up of the electric oil pump

As shown in Figure 3, the electric oil pump starts at the 3rd second (reaching the rated speed), the oil pump outlet starts to establish the pressure and reaches the peak value of 1.28 in the 16th second. The whole pressure building process takes 13 seconds and finally stabilizes at 1.15. Since the oil pipeline in the system has a certain volume, and the pipeline is basically empty when the system is stopped, during the start-up of the electric oil pump, there is a process in which the oil is filled to the pipelines of the each equipment. So from the electric oil pump there are different time delays between the pressure establishment of the oil pump to the pressure establishment of each equipment node. Among them, the turbocharger is the farthest from the oil pump and has the highest spatial position, so its delay of establishing the pressure is also the longest, about 14 seconds. After 35 seconds, the oil pressure of the whole system tends to be stable, and a stable oil pressure difference is established between the inlets of the equipment. The pressure difference is determined by the relative height of each equipment and the flow resistance of the pipeline. The flow resistance between the equipment of the same height is mainly derived from the oil filter. For example, the filter between the outlet of the electric oil pump and the main oil pipe will cause a pressure drop of about 0.18. It is known after the analysis that the response performance of the lubricating oil system is related to the establishment time of the oil pump outlet pressure, the installation position of the equipment, the flow resistance of the pipeline, etc. Reducing the time for pressure establishment of the oil pump can directly improve the system's response speed and reduce the flow resistance of the inlet filter of each oil equipment can significantly reduce the flow resistance of the oil and increase the pressure of the imported oil.

\subsection{Dynamic simulation of the starting process of turbine Oil Pump}

In order to study the start-up characteristics of the turbine oil pump and the dynamic changes of the oil pressure of each key equipment during the process from the low working condition to the high working condition, the process that the turbine oil pump starts and then is put into use when the electric oil pump is working alone is selected as a simulation experiment. Since the speed of the turbine oil pump does not change in a linear process during the actual test, this model selects the auxiliary turbine module, the steam turbine rotor module, the rotating shaft module nonlinear interpolation module, etc. to simulate the nonlinear 
acceleration process of the turbine oil pump. When the operating state of the system changes, the speed of oil pump will also fluctuate accordingly.

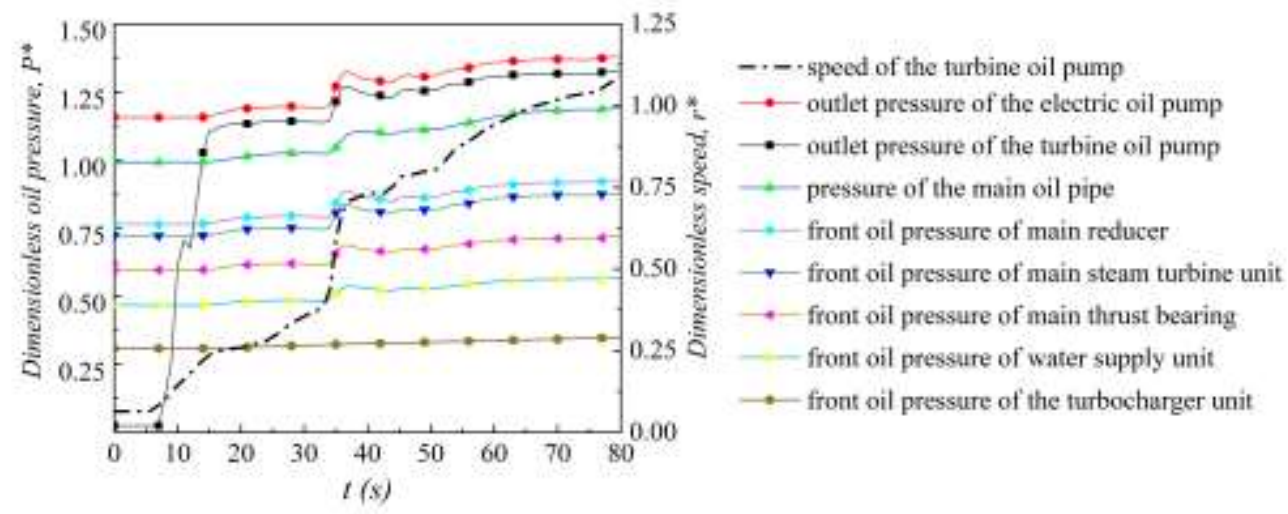

Fig 4: Changes in system parameters during start-up of the turbine oil pump

As shown in Figure 4, the turbine oil pump starts to rotate at the 7th second. At the 8th second, the oil pump outlet starts to establish pressure. At the 60th second, the outlet pressure of the oil pump reaches 1.30 and tends to stabilize. At this time, the speed of the turbine oil pump is about 0.9 , and the entire process takes about 53 seconds. After the 60th second, the outlet pressure of the oil pump does not change significantly with the increase of the speed. The main reason for this phenomenon is that the oil pump is equipped with a pressure relief valve. When the outlet pressure is higher than the rated value, part of the oil will return to the oil tank through the pressure relief valve, thus ensuring the stability of the outlet pressure of the oil pump. Since the turbine oil pump is started under the low working condition of the system, the stable oil pressure has been established in the system, so the oil pressure of the each key node of the system does not fluctuate greatly during the start-up process of the turbine oil pump. At the 35th second, the speed of the turbine oil pump increases sharply, which causes the oil pressure at the outlet of the turbine oil pump and the oil pressure at each node of the system to increase significantly. Due to the bidirectional transmission characteristics of the pressure in the fluid network, the electric oil pump in stable operation appears the phenomenon that the export pressure rises synchronously.

\subsection{Simulation of oil temperature disturbance}

In order to study the change of the oil temperature during the dynamic operation of the system and the dynamic response of the oil temperature regulating valve, the oil temperature at the outlet of the oil cooler and the opening value of the temperature control valve during the stable operation of the lubricating oil system are selected as objects to expand the analysis. As shown in Fig. 5, when outlet oil's temperature of the cooler exceeds 1.04 , the oil temperature regulating valve starts to operate, and when outlet oil's temperature of the cooler is lower than 0.91 it stops working. The system's oil temperature is stable within the range of 0.86 to 1.09 under the regulating action of the regulating valve to meet the oil requirements of the power unit.

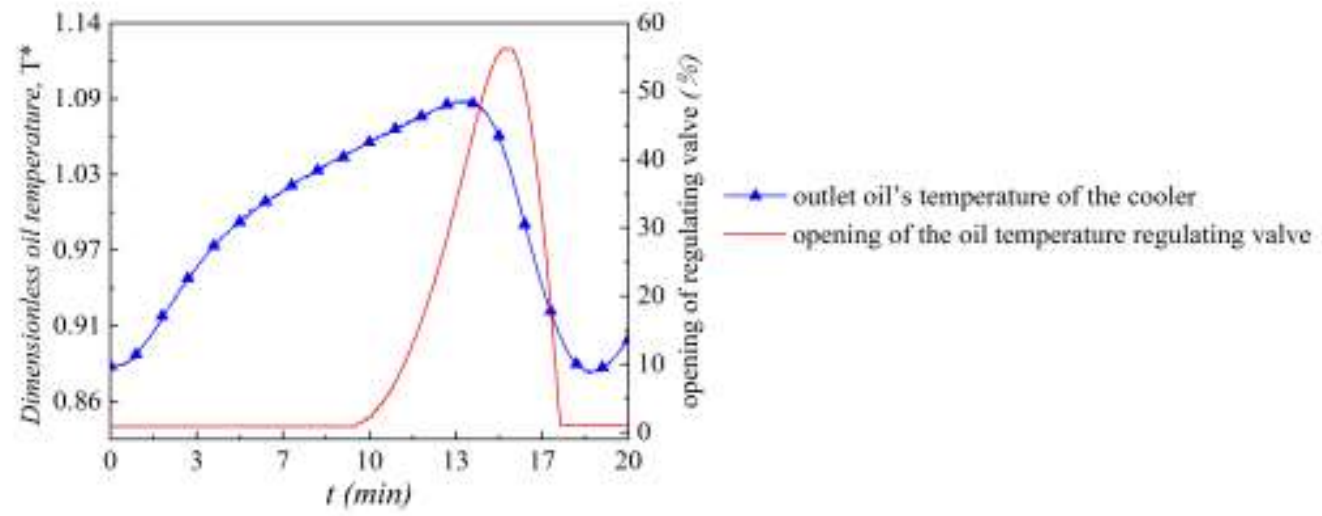

Fig 5: Changes in oil temperature and opening of the regulating valve during a steady state process

\section{Conclusion}


Based on the modeling method of complex fluid network system, this paper modularizes the lubricating oil system of the marine power plant and applies the SimuWorks simulation platform to simulate the typical dynamic process of the system. The main research results are as follows:

(1) By studying the actual power plant, the influence of key factors such as equipment scale and relative position of the equipment on the operation of the system is considered. By introducing the energy balance equation, the problem of heat exchange between the oil and pipe wall and pipe wall and the environment are considered during the operation of the system. The continuity equation, the momentum conservation equation and the energy balance equation are used jointly to realize the coupling calculation of oil flow and heat transfer.

(2) According to the simulation calculation results, the response performance of the lubricating oil system of the marine power plant is closely related to the pressure building capacity of the oil pump, the spatial position of the equipment, the resistance of the oil flow, etc. The reduction of the pressure of the oil pump can directly improve the response speed of the system. And the reduction of the flow resistance of the filter at the inlet of each oil equipment can significantly improve the system's working performance.

(3) The component model developed based on SimuWorks platform has good versatility and openness, can be used for analysis of flow and heat transfer characteristics of various thermal fluid network systems. It can provide technical support for system control strategy optimization, fault diagnosis and retrofit design, etc and have certain engineering value.

\section{References}

[1] YAN Xinghui , GUO Yingqing, YIN Kai, et al. Modeling simulation and optimization of oil system based on MATLAB/Simulink[J]. Journal of Aerospace Power, 2017(3).

[2] REN Guo-zhe , LIU Zhenxia, ZHU Pengfei, et al. Calculation on Heat-Fluid Coupling of Pipes in Aero-Engine Lubrication System[J]. Journal of Propulsion Technology, 2016, 37(3):504-510.

[3] BAO Han, WANG Xinge, YE Kunwu, Anlysis on Pressure of Lubricating Oil System Based on Flowmaster[J]. Mechanical Research and Application , 2017(04):37-39.

[4] Tan W Z, Huang Q W , Zhang C , et al. Study on modeling of marine propulsion system coupled with bearing hydrodynamic lubrication[C]// 2015 International Conference on Transportation Information and Safety (ICTIS). IEEE, 2015.

[5] Chun S M. Network analysis of an engine lubrication system[J]. Tribology International, 2003, 36(8):609-617.

[6] Moussa Nahim H , Younes R, Shraim H , et al. Modeling with Fault Integration of the Cooling and the Lubricating Systems in Marine Diesel Engine: Experimental validation[J]. IFAC-PapersOnLine, 2016, 49(11):570-575.

[7] SU Lichao, LIU Zhenxia, LV Yaguo . Simulation and calculation of aeroengine oil system pressure[J]. Science Technology and Engineering, 2012(1):97-101.

[8] Ni He . Modeling and Technology State Assessment of Complex Thermal System[M]. 2016:89-90.

[9] Ni He, Cheng Gang, Sun Fengrui. Simple Modular Modeling Method of Thermo-fluid Network [J]. Journal of System Simulation, 2009, 21(12): 3536-3541.

[10] CAI Ruizhong, YU Shengfang, Wang Jianfu, et al. An Improved Numerical Calculation Method of Fluid Network for Real Time Simulation of Fossil Power Plant[J]. Science \& Technology Review , 2007, 25(8).

[11] Huang Shengqin, LIU Zhenxia, LV Yaguo, et al. Numerical Simulation of Flow and Heat Transfer on Lubricant Supplying and Returning Flow Path of an Aero-Engine Lubrication System[J]. Computer Simulation , 2008, 25(6).

[12] ZHANG Shuyuan . Research on lubrication system of ship transmission based on fluid network algorithm[J]. Ship Science and Technology , 2017(14):137-139.

[13] NI He, XIAO Hang, ZENG Fanming, et al. Segmented Lumped Parameter Modeling of Steam Turbine Based on Cascad Calculation[J]. Journal of System Simulation , 2014, 26(2):294-299. 